\title{
Virtual Screening Approaches in Identification of Bioactive Compounds Akin to Delphinidin as Potential HER2 Inhibitors for the Treatment of Breast Cancer
}

\author{
Kavisha Patidar $^{1}$,Aruna Deshmukh ${ }^{1}$, Srinivas Bandaru $^{3}$, Chandana Lakkaraju ${ }^{3}$, \\ Amandeep Girdhar ${ }^{1}$, Gutlapalli VR ${ }^{1}$, Tushar Banerjee ${ }^{2}$, Anuraj Nayarisseri ${ }^{1,4}$, \\ Sanjeev Kumar Singh**
}

\begin{abstract}
Small molecule tyrosine kinase inhibitors targeting HER 2 receptors have emerged as an important therapeutic approach in inhibition of downstream proliferation and survival signals for the treatment of breast cancers. Recent drug discovery efforts have demonstrated that naturally occurring polyphenolic compounds like delphinidin have potential to inhibit proliferation and promote apoptosis of breast cancer cells by targeting HER2 receptors. While delphinidin may thus reduce tumour size, it is associated with serious side effects like dysphonia. Owing to the narrow therapeutic window of delphinidin, the present study aimed to identify high affinity compounds targeting HER2 with safer pharmacological profiles than delphinidin through virtual screening approaches. Delphinidin served as the query parent for identification of structurally similar compounds by Tanimoto-based similarity searching with a threshold of $95 \%$ against the PubChem database. The compounds retrieved were further subjected to Lipinski and Verber's filters to obtain drug like agents, then further filtered by diversity based screens with a cut off of 0.6 . The compound with Pubchem ID: 91596862 was identified to have higher affinity than its parent. In addition it also proved to be non-toxic with a better ADMET profile and higher kinase activity. The compound identified in the study can be put to further in vitro drug testing to complement the present study.
\end{abstract}

Keywords: Delphinidin - HER2 - breast cancer - virtual screening - molecular docking

Asian Pac J Cancer Prev, 17 (4), 2291-2295

\section{Introduction}

Metastatic breast cancer is an irremediable disease with a 2- to 3-year median overall survival time (Park et al., 2015). Breast cancer is the one of the well known cause of death in women worldwide and among the leading cause of cancer in women in India (Desai et al., 2000). According to GLOBOCAN database of the International Agency for Research on Cancer (IARC) that the frequency of breast cancer in women living in developing regions is steeply increasing (Parkin et al., 2001). The human epidermal growth factor receptor 2 (HER2) gene, also known as neu and c-erbB-2, which is placed on chromosome 17q21 which encodes a 185-kd transmembrane glycoprotein receptor protein (p185HER2) on breast cells (Vogel et al., 2002) has been significantly associated with breast carcinogenesis.

It has huge similarity with other members of the EGFR family, which encompasses ErbB-1, ErbB-3 and
ErbB-4. Mechanisms behind HER-2 overexpression include gene amplification and increased transcription, which then sequentially leads to enhance protein turnover (Vaidyanathan et al., 2010). ErbB-2 can both homodimerize and heterodimerize with other members of the EGFR family and initiates a series of signal transduction pathways via the MAPK and PI3K pathways (Vaidyanathan et al., 2010).

Gene amplification is known to be one of the significant mechanisms responsible for HER-2 overexpression (Vaidyanathan et al., 2010). Approaches in disruption of Her2 dimerization have now surfaced as an important strategy in treatment of breast cancer. Although the availability of potent HER2-targeted therapies, drug discovery efforts continue to find out supplementary agents that may inhibit breast cancer cell growth, particularly examining naturally drugs that may be useful in multiple subtypes of breast cancer (Desai et al., 2000).

Recent drug discovery efforts have demonstrated that

${ }^{I}$ In silico Research Laboratory, Eminent Biosciences, ${ }^{2}$ School of Life Science, Devi Ahilya University, Khandwa Road, Indore, Madhya Pradesh, ${ }^{3}$ Institute of Genetics and Hospital for Genetic Diseases, Osmania University, Hyderabad, ${ }^{4}$ Computer Aided Drug Designing and Molecular Modeling Lab, Department of Bioinformatics, Alagappa University, Karaikudi, Tamil Nadu, India *For correspondence: skysanjeev@gmail.com 
the naturally occurring polyphenolic compounds have potential to inhibit proliferation and promote apoptosis of breast cancer cells (Desai et al., 2000). A diphenylpropanebased polyphenolic ring structure compound, delphinidin that carries a positive charge in its central ring has been shown to inhibit proliferation and promote apoptosis in many different cancer models including colon, uterine, breast, and prostate (Hafeez et al., 2008; Chanda et al., 2015). Though, delphinidin has shown to have tumor reduction (Ozbay et al., 2011) however has been associated with serious side effects like dysphonia. Individuals taking delphinidin reported dysphonia to the FDA. A total of 2 delphinidin 3-glucoside drug adverse event reaction reports were made with the FDA during 2004-2013 (Gimanez et al., 2016).Therefore, in the view of above and considering the potential side effects of Delphinidin, we sought to identify a novel compound bestowed with higher affinity against Her2protein and possibly having commendable potential to inhibit proliferation and promote apoptosis with reduced side effects.

\section{Materials and Methods}

\section{Selection of inhibitors}

Delphinidin belonging to compound class of diphenyl propane served as query molecule for linear finger print similarity search.

\section{Preparation of protein and compounds}

The crystal structure of ErbB2-pertuzumab complex was from Protein Data Bank (PDB) with PDB ID: 1S78 (Figure 1) (Franklin et al., 2004). The structure was downloaded in .pdb format and was further prepared for docking process. The protein was prepared using the PrepWiz module of Schrodinger suite (Bandaru et al., 2014). In the preparation procedure, the protein was first preprocessed by assigning the bond orders and hydrogen, creating zero order bonds to metals and adding disulphide bonds.

The missing side chains and loops were filled using Prime Module of Schrodinger. Further all the water molecules were deleted beyond $5 \AA$ from hetero groups. Once the protein structure was preprocessed, $\mathrm{H}$ bonds were assigned which was followed by energy minimization by OPLS 2005 force field. The final structure obtained was saved in.pdb format for further studies. All the ligands were optimized through OPLS 2005 force field algorithm (Jorgensen et al., 2005) embedded in the LigPrep module of Schrödinger suite, 2013 (Schrodinger. LLC, New York, NY). The ionizations of the ligand were retained at the original state and were further desalted. The structures thus optimized were saved in .sdf format for docking procedures (Kelotra et al., 2014).

\section{Structure similarity search}

Similarity search was supervised by binary finger print based Tanimoto similarity equation to retrieve compounds similar to Delphinidin with similarity threshold of $95 \%$ against NCBI's Pubchem compound database.
Molecular docking program Molegro Virtual Docker (MVD, 2010.4.0.0) which includes highly efficient PLP (Sahila et al., 2015, Babitha et al., 2015) and MolDock scoring function (Thomsen et al., 2006) provided flexible platform for molecular docking (Dunna et al., 2015). The optimized structures of delphinidin were docked into the binding cleft of Her2 protein. Docking parameters were set to $0.20 \AA$ as grid resolution, maximum iteration of 1500 and maximum population size of 50. Energy minimization and hydrogen bonds were optimized after the docking. Simplex evolution was set at maximum steps of 300 with neighborhood distance factor of 1 . Binding affinity and interactions of inhibitor with protein was evaluated on the basis of the internal ES (Electrostatic Interaction), internal hydrogen bond interactions and sp2-sp2 torsions. Post docked ligand-receptor complex energy was minimized using the Nelder Mead Simplex Minimization approach (using non-grid force field and $\mathrm{H}$ bond directionality) (Nelder et al, 1965).

Toxicity screening and bioactivity prediction of compounds

All the similar compounds retrieved were screened for its drug ability by lipinksi filters. The toxicity screening was achieved by using LAZAR toxicity prediction server (Maunz et al., 2013). Biological activity of the ligands was predicted using Molinspiration webserver (C) Molinspiration Cheminformatics 2014). LC 50 was predicted using T.E.S.T. Version 4.1 (2012, U.S. Environmental Protection Agency) software. The complete ADMET properties was calculated using admetSAR (Cheng et al., 2012)

\section{Software, Suites and Webservers used}

For virtual screening Pubchem database was used to search and prepare library of similar chemical compounds. All the chemical structures were drawn in MarvinSketch

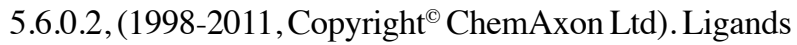
were optimized with LigPrep module of Schrodinger suite 2013. Protein was processed and refined with

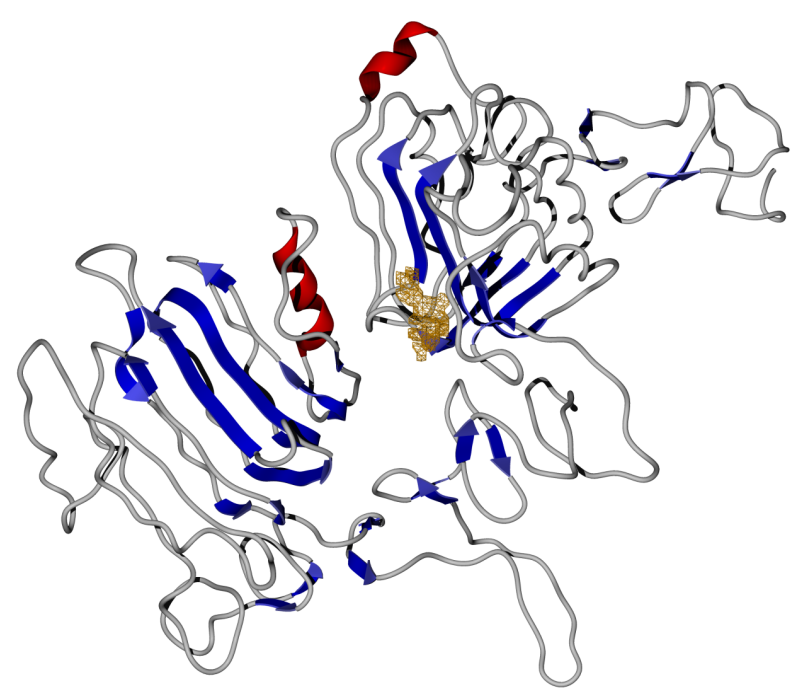

Figure 1. Secondary Structure Representation of X-ray Crystal Structure of the Extracellular Domain of the Human Epidermal Growth Factor Receptor 2 (ErbB2 or HER2). The orange shaded region is the inhibitor binding site 
Virtual Screening for Bioactive Compounds akin to Delphinidin as Potential HER2 Inhibitors for Breast Cancer Treatment Table 1. Affinity (Rerank) Scores of the Best Docking Compounds

\begin{tabular}{|c|c|c|c|c|}
\hline & Pubcid: 91596862 & $\frac{\text { Pubcid: }}{87069394}$ & $\frac{\text { Pubcid: }}{49870418}$ & Delphinidin \\
\hline Energy overview: Descriptors & Rerank Score & Rerank Score & Rerank Score & Rerank Score \\
\hline Total Energy & -78.492 & -73.779 & -72.482 & -70.56 \\
\hline External Ligand interactions & -89.07 & -84.708 & -81.421 & -78.025 \\
\hline Protein - Ligand interactions & -89.07 & -84.708 & -81.421 & -78.025 \\
\hline Steric (by PLP) & -73.728 & -70.421 & -66.421 & -57.674 \\
\hline Steric (by LJ12-6) & -25.754 & -22.391 & -19.214 & -14.43 \\
\hline Hydrogen bonds & -7.92 & -6.896 & -3.418 & -2.92 \\
\hline Internal Ligand interactions & -14.445 & -13.665 & -8.564 & -6.001 \\
\hline Torsional strain & 10.226 & 8.366 & 6.444 & 3.167 \\
\hline Steric (by PLP) & -4.6 & -2.3 & -1.3 & 0.94 \\
\hline
\end{tabular}

Table 2. ADMET Profile sCalculated for Best Docked Compound from Each Dataset by AdmetSAR

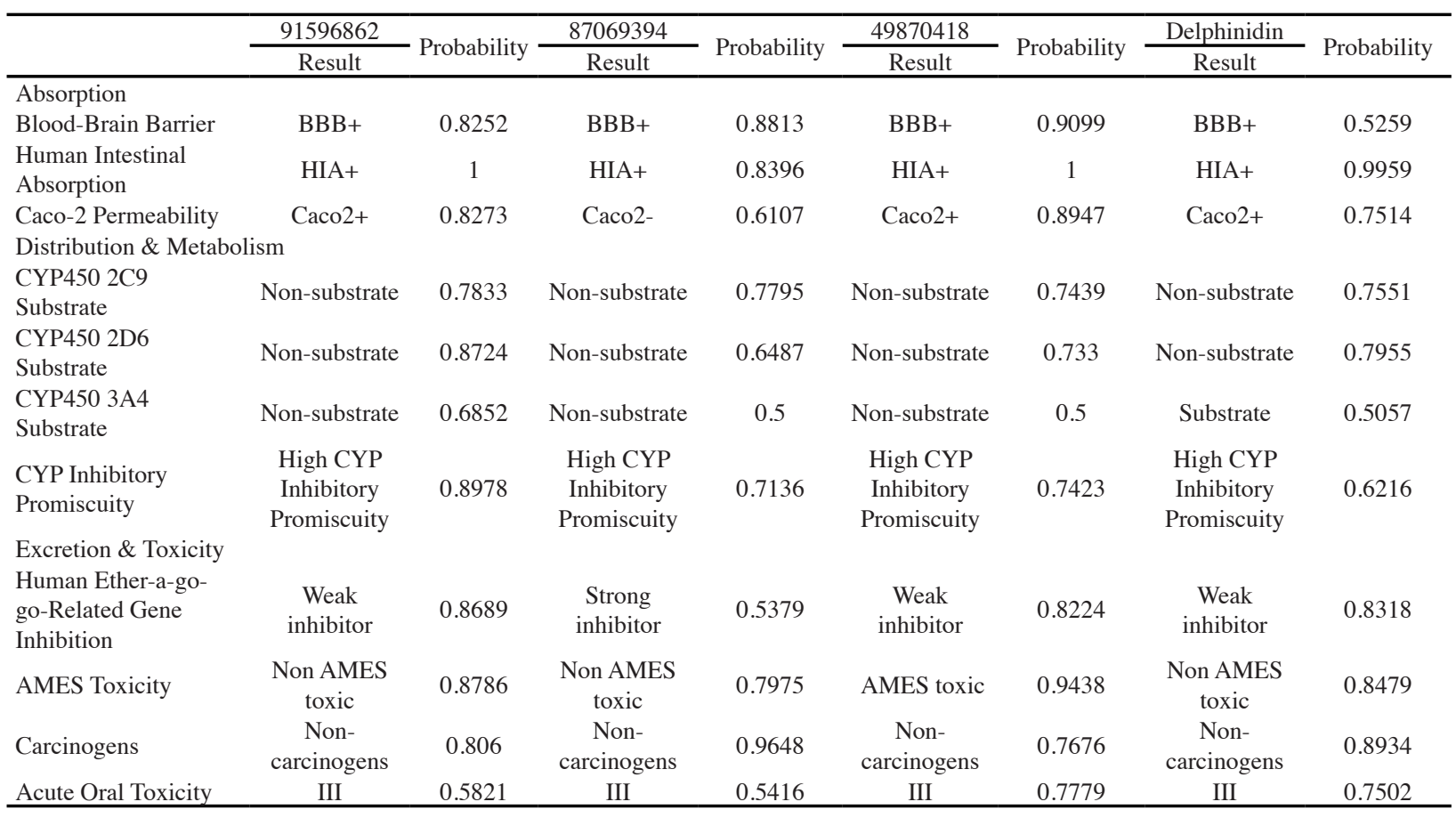

protein preparation wizard of Schrodinger suite 2013 (Schrodinger. LLC, 2009, New York, NY).

Flexible molecular docking of the compounds with target was completed using the Molegro Virtual Docker 2010.4.0.0. Accelrys Discovery Studio ${ }^{\circledR}$ Visualizer 3.5.0.12158 (Copyright(C) 2005-12, Accelrys Software Inc.) was used for molecular visualizations. LAZAR online server was employed to predict in silico toxicity. T.E.S.T software (2012, U.S. Environmental Protection Agency) and the Molinspiration web server (C) Molinspiration Cheminformatics 2014) were respectively used for predicting LC50 and bioactivity of the compound. ADMET profiles were calculated using admeSAR (Laboratory of Molecular Modeling and Design. Copyright ${ }^{\circledR}$ 2012, East China University of Science and Technology, Shanghai Key Laboratory for New Drug Design,)

\section{Pharmacophoric mapping}

Pharmacophoric mapping which involves ligand interaction patterns, hydrogen bond interaction, hydrophobic interactions was evaluated using Accelrys Discovery Studio 3.5 DS Visualizer.

\section{Results and Discussion}

A total of 550 similar structures were identified against Delphinidin query through linear finger based tanimoto search metric. In order to obtain the drug like compounds, the compound library obtained from linear finger print based search was further screened to retrieve compounds which followed Lipinki et al. (2004) as well as Veber et al (2002) rules. A total of 114 compounds out of library of 550 compounds that passed Lipinski filters were further subjected to diversity based screens in order to retrieve non-redundant compounds with non-overlapping chemical features. The diversity based screens revealed 35 structures chemically diverse to each other. These 35 compounds were further subjected to structure based virtual screening through molecular docking approaches. The complete virtual screening process is shown in Figure 2.

The top three compounds obtained from extensive ligand and structure based screening were compound with Pub Cid: 91596862 (Figure 3a), followed by compound with PubCid: 87069394 (Figure 3b) and compound with PubCid : 49870418 (Figure 3c). It is interesting to note that all the three retrieved through virtual screening approaches 
Table 3. Predicted LC50 Values and Bioactivity of Compounds

\begin{tabular}{|c|c|c|c|c|c|c|c|}
\hline \multicolumn{2}{|c|}{ Lethal Dose Concentration } & \multicolumn{6}{|c|}{ Bioactivity } \\
\hline $\begin{array}{c}\text { Compounds with best } \\
\text { docking profiles }\end{array}$ & LC50 (96 hr) mg/L & $\begin{array}{l}\text { GPCR } \\
\text { ligand }\end{array}$ & $\begin{array}{c}\text { Ion channel } \\
\text { modulator }\end{array}$ & $\begin{array}{l}\text { Kinase } \\
\text { inhibitor }\end{array}$ & $\begin{array}{c}\text { Nuclear receptor } \\
\text { ligand }\end{array}$ & $\begin{array}{l}\text { Protease } \\
\text { inhibitor }\end{array}$ & $\begin{array}{l}\text { Enzyme } \\
\text { inhibitor }\end{array}$ \\
\hline PubCid: 91596862 & 0.14 & 0.03 & 0.06 & -0.1 & 0.32 & -0.2 & 0.13 \\
\hline PubCid:87069394 & 0.18 & 0.25 & -0.11 & -0.13 & 0.12 & 0.14 & 0.15 \\
\hline PubCid:49870418 & 0.29 & -0.01 & 0.04 & -0.12 & 0.23 & -0.21 & 0.06 \\
\hline Delphinidin & 0.34 & -0.15 & 0.05 & -0.14 & 0.43 & -0.08 & 0.16 \\
\hline
\end{tabular}

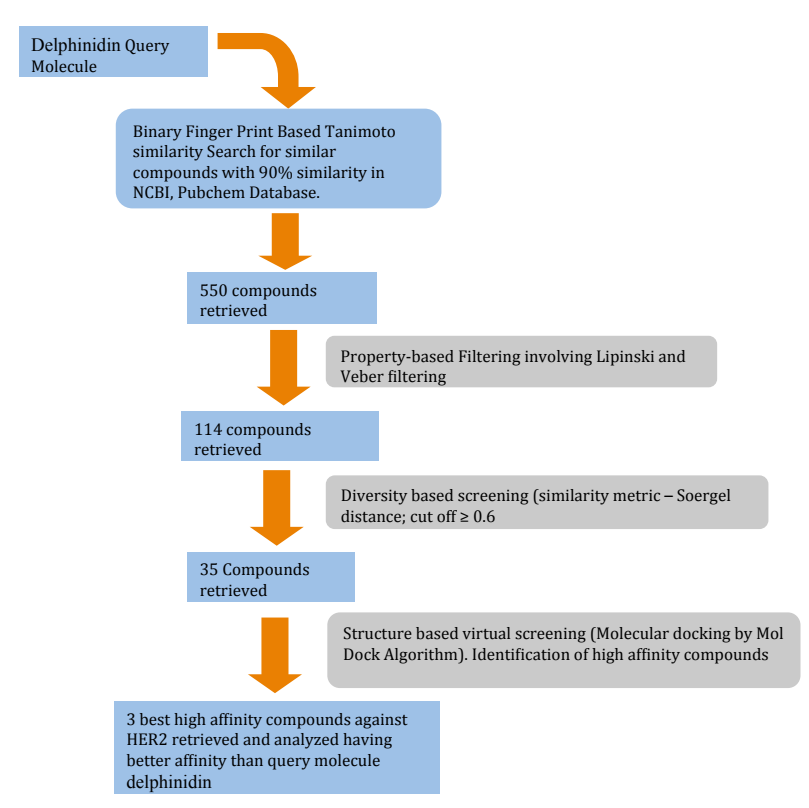

Figure 2. Ligand and Structure Based Screening Employed in the Study<smiles>CCOc1cc2cc(O)cc(O)c2cc1-c1cc(OC)c(O)c(OC)c1</smiles><smiles>COc1cc2c(O)cc(O)cc2nc1-c1cc(OC)c(O)c(OC)c1</smiles><smiles>COC1=CC(OC)=C2OC(c3cc(O)c(O)c(OC)c3)=C(O)C=C2C=C1O</smiles>

Figure 3. Chemical structure of compounds (A) Pub Cid: 91596862, (B) PubCid: 87069394 (C) PubCid : 49870418 and (D) Delphinidin

had higher affinity than Delphinidin (Figure 3d). The detailed affinity scoring involving different interactions contributing to final rerank score is shown in Table 1.

In the further study we pursued to find the rationale behind the better binding affinity PubCid: 91596862 against HER2. Considering different interactions we observed that the superior affinity of compound PubCid: 91596862 than Delphinidin can be attributed to its excellent interaction profile especially in terms of electrostatic and $\mathrm{H}$-bonding interactions. Apparent from the docking profile of compound PubCid: 91596862 values of descriptors of external ligand interactions contribute 6.16 folds higher stability than internal ligand interactions. Further external ligand interactions were
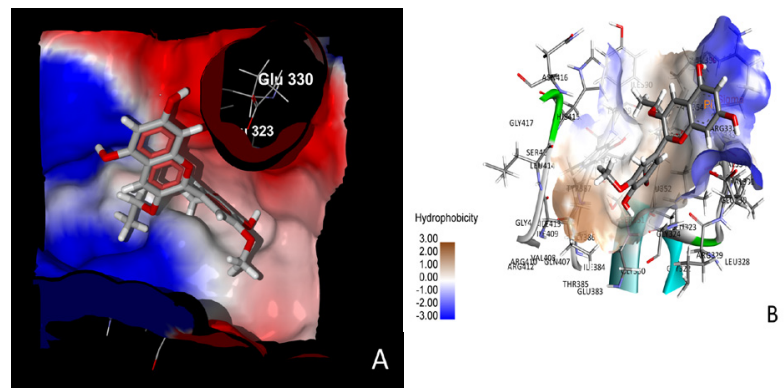

Figure 5. (A)Electrostatic Interactions of Pubcid: 91596862 with HER2. (B) The binding site of HER 2 Harboring Compound Pubcid: 91596862 is shown with Hydrophobic Intensities. The hydrophobic intensities of the binding site ranges from -3.00 (least hydrophobic area - blue shade) to 3.00 (highly hydrophobic area -brown shade)

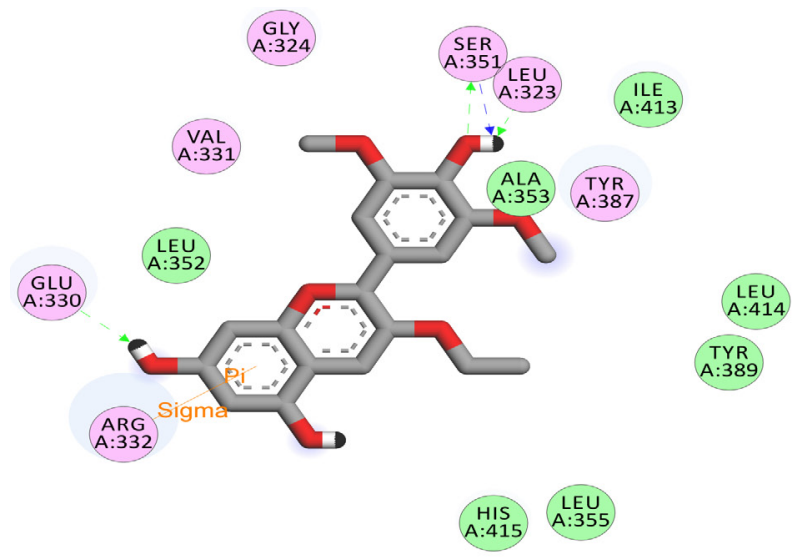

Figure 4. Interactions of Pubcid: 91596862 in HER2

Receptor. Residues circled in green participate in van der Waals interaction with the ligand while residues in pink forms electrostatic interactions

stabilized mostly by stearic energy guided by Piece wise linear potentials and Lenard Jones potentials. While in internal ligand interactions, the torsional strain contributes for the stability of the ligand receptor interactions.

The ADMET profiles (Table 2) of the three best docked compounds along with Delphinidin revealed that compound Pubcid: 91596862 was better compound and most likely drug like compared to its parent compound delphinidin. While compound PubCid: 87069394 was also predicted to be safe but 49870418 proved to be ames toxic. In addition, the predicted bioactivity (Table 3 ) as well as the LC 50 values of compound Pubcid: 91596862 was quite appreciable. The LC 50 value of Pubcid: 91596862 at 96 hour interval was predicted to be 2.42 folds superior to its parent compound Delphinidin. In addition all the three best docked compounds identified showed enhanced bioactivity, but it was Pubcid: 91596862 which showed 
Virtual Screening for Bioactive Compounds akin to Delphinidin as Potential HER2 Inhibitors for Breast Cancer Treatment

best score for kinase inhibition activity, providing a clue for target specificity. The pharmacological profiles of the entire three best docked compounds and parent compound Delphinidin were although appreciable, but it was compound Pubcid: 91596862 which showed best amongst all the compounds studied and therefore it was further analyzed for pharmacophoric mappings.

Comprehensively shown in Figure 4, the compound Pubcid: 91596862 demonstrates van der Waals interactions with Ile 413, Ala 353, Leu 414, Tyr 389 Leu 355 His 415 and Leu 352 and electrostatic interactions with Arg 332, Glu 330, Val 331, Gly 324, Ser 351, Leu 323 and Tyr 387. compound Pubcid: 91596862 is a hydrogen bond donor to electrostatic residue Ser 351 and acceptor from Ser 351, Leu 323 and Glu 330. In addition, sigma interactions are seen between ligand and Arg 332. Electrostatic and hydrophobic interactions of compound Pubcid: 91596862 in the site are shown in Figure 5a and Figure 5 b respectively.

In conclusion, the narrow therapeutic window of available HER2 inhibitors especially Delphinidin necessitates an urgent need to develop new drugs treatment of breast cancer. Therefore in the given view we identified compounds derived from virtual screening process with optimal pharmacological profile. In the study, compound PubCid: 91596862 akin to delphindin demonstrated drug like properties endowed with higher binding affinity, least toxicity and optimal bioactivity. The compound identified in the study can be further complemented by In vitro drug testing.

\section{References}

Babitha PP, Sahila MM, Srinivas Bandaru, et al (2015) Molecular docking and pharmacological investigations of rivastigminefluoxetine and coumarin-tacrine hybrids against acetyl choline esterase. Bioinformation, 11, 378-86.

Bandaru S, Ponnala D, Lakkaraju C, et al (2014). Identification of high affinity non-peptidic small molecule inhibitors of MDM2-p53 interactions through structure-based virtual screening strategies. Asian Pac J Cancer Prev, 16, 3759-65.

Chanda S, Anuradha N, Srinivas B, et al (2015). An in silico approach for identification of novel inhibitors as a potential therapeutics targeting hiv-1 viral infectivity factor. Curr Top Med Chem, 15, 65-72.

Cheng F, Li W, Zhou Y, et al (2012). AdmetSAR: a comprehensive source and free tool for assessment of chemical ADMET properties. J Chem Inf Model, 52, 3099-105.

Desai SB, Moonim MT, Amarinder KG, et al (2000). Hormone receptor status of breast cancer in India: a study of 798 tumours. Breast, 9, 267-70.

Dunna NR, Kandula V, Girdhar A, et al (2015). High Affinity Pharmacological Profiling Of Dual Inhibitors Targeting Ret And Vegfr2 In Inhibition Of Kinase And Angiogeneis Events In Medullary Thyroid Carcinoma. Asian Pac J Cancer Prev, 16, 7089-795.

Franklin, MC, Carey KD, Vajdos FF, et al (2004). Insights into ErbB signaling from the structure of the ErbB2-pertuzumab complex. Cancer cell, 5, 317-28.

Gimanez MA, Drago SR, Bassett MN, et al (2016). Nutritional improvement of corn pasta-like product with broad bean (Vicia faba) and quinoa (Chenopodium quinoa). Food Chem, 199, 150-56.

Hafeez BB, Siddiqui IA, Asim M et al (2008). A dietary anthocyanidin delphinidin induces apoptosis of human prostate cancer PC 3 cells In vitro and in vivo: involvement of nuclear factor- $\varkappa \mathrm{B}$ signaling. Cancer Res, 68, 8564-72.

Jorgensen WL, Tirado-RJ (2005). Potential energy functions for atomic-level simulations of water and organic and biomolecular systems. Proc Natl Acad Sci U S A, 102, 6665-70.

Kelotra S, Jain M, Kelotra A, et al (2014). An in silico appraisal to identify high affinity anti-apoptotic synthetic tetrapeptide inhibitors targeting the mammalian caspase 3 enzyme. Asian Pac J Cancer Prev, 15, 10137-42.

Lipinski CA, et al (2004). Lead-and drug-like compounds: the rule-of-five revolution. Drug Discov Today Technol, 1,337-41.

Maunz A, Gütlein M, Rautenberg M, et al (2013). Lazar: a modular predictive toxicology framework. Front Pharmacol, 4,38

Nelder JA, Mead R (1965). A simplex method for function minimization. Comput J, 7, 308-13.

Ozbay T, Nahta R (2011). Delphinidin inhibits HER2 and Erk1/2 signaling and suppresses growth of HER2-overexpressing and triple negative breast cancer cell lines. Breast Cancer (Auckl), 5, 143-54.

Park Y, Hyun-Tae S, Hae HJ, et al (2015). Role of HER2 mutations in refractory metastatic breast cancers: targeted sequencing results in patients with refractory breast cancer. Oncotarget, 6, 32027-38.

Parkin DM, Bray F, Ferlay J, et al (2001). Estimating the world cancer burden: Globocan 2000. Int J Cancer, 94, 153-56.

Sahila MM, Babitha PP, Srinivas Bandaru, et al (2015) Molecular docking based screening of GABA (A) receptor inhibitors from plant derivatives. Bioinformation, 11, 280-89.

Thomsen R, Christensen MH (2006). MolDock: a new technique for high-accuracy molecular docking. J Med Chem, 49, 3315-21.

Vaidyanathan K, Kumar P, Reddy CO, et al (2010). ErbB2 expression and its association with other biological parameters of breast cancer among Indian women. Indian J Cancer, 47, 8-15.

Veber DF, Johnson SR, Cheng HY, et al (2002). Molecular properties that influence the oral bioavailability of drug candidates. J Med Chem, 45, 2615-23.

Vogel CL, Cobleigh MA, Tripathy D, et al (2002). Efficacy and safety of trastuzumab as a single agent in first-line treatment of HER2-overexpressing metastatic breast cancer. J Clin Oncol, 20, 719-26. 